\title{
Certified Diabetes Educator: An Important Aid in Improving Patient Care in Diabetes
}

\author{
Ameya Joshi ${ }^{1}$ \\ R. D. Sana N. Shaikh² \\ ${ }^{1}$ Department of Endocrinology, Bhaktivedanta Hospital and INDEAN \\ Clinics Mumbai, Maharashtra, India \\ 2Nutritionist and Diabetes Educator, INDEAN Clinics Mumbai, \\ Maharashtra, India
}

Address for correspondence Ameya Joshi, MD, DM, 202, C-wing, AVIRAHI CHS, Prem Nagar, Near Shanti Garden, Mira Road East, Thane 401107, Maharashtra, India (e-mail: ameyaable@gmail.com).
Diabetes mellitus is a chronic disease in which education plays a vital part. However, in India that is overburdened with diabetes has short of diabetes educators. Care for diabetes is limited by the adverse physician-to-patient ratio and lack of trained paramedical personnel. The following review focuses on role of diabetes educators in diabetes care and their current status in India.

- National Diabetes

Education Program

\section{Introduction}

Diabetes mellitus (DM), an overgrowing disease, is affecting 72.9 million of people worldwide. The number of patients keeps on increasing and so does the pressure on the health care professionals. An emerging field came into being called certified diabetes educator (CDE) ${ }^{1}$

Physician-to-patient ratio in India is $1: 1,800$. The government has recommended at least 1:1,000 ratio. Owing to the inadequate workforce, it becomes difficult to manage patients or even give them a preventive or behavior changing session. It is where CDE plays a very important role in educating such patients and helping them manage the condition.

Currently, there are 780 members in the Association of Diabetes Educator (ADE), and most of them are CDE except for physicians. One of their eligibility criteria is that a person should be a CDE with adequate experience. The most common barrier stands the lack of time, collaboration, and motivation., ${ }^{2,3}$

\section{History}

DM is a chronic lifestyle ailment, and lifestyle modification remains the cornerstone of DM management. DM management also involves educating patients about the disease itself, treatment targets, monitoring and follow-ups, hypoglycemia, and footcare and sensitization to complications. Elliott P. Joslin ${ }^{4}$ believed that patient education is important. Dr. Joslin was a true and devoted teacher as he educated patients and their family members. He also taught it to the health care professionals.
In 1957, Dr. Joslin realized of creating a model of diabetes treatment and education center. He opened the Diabetes Treatment Unit (DTU). It had classrooms, kitchen, and gymnasium. Patients used to stay for 1 week to learn as much as possible on how to live with diabetes. He had physicians who took classes, but he himself also took lectures sometimes., ${ }^{4,5}$

CDE is a health care professional who is specialized in managing and preventing diabetes as he/she are trained well into developing comprehensive knowledge and skills. They form a link between physicians and patients. They modify patients' behavior and teach them self-management. They promote behavior changes by

- Assessment of the condition

- Setting a goal

- Planning the management

- Implementing

- Evaluating the behavior and documenting it

\section{Eligibility}

To become a CDE in the Unites States, it is necessary to meet the eligibility requirements set by the National Certification Board for Diabetes Educators (NCBDE).

NCBDE has set two eligibility requirements:

- Eligible health care professional, that is, nurse, dietitian, pharmacist, occupational or physical therapist, etc.

- Professional practice experience with a minimum of 1,000 hours of diabetes self-management education experience, 
with $40 \%$ of those hours accrued in the most recent year preceding application. ${ }^{6}$

In India there is no regulatory body defining standards for CDE.

\section{National Diabetes Educator Program}

The National Diabetes Educator Program (NDEP) is a program that was developed with an objective of creating CDE. CDEs are specialized and certified to educate patients about aspects of DM management. This program is backed by Dr. Mohan Diabetes Education Academy (DMDEA), a unit of Dr. Mohan Diabetes Specialties Centre and the Indian Association of Diabetes Educators (IADE).

\section{Program Structure}

- This course is being conducted in 140 pre-selected cities/ centers.

- Each center enrolls more than 10 candidates.

- The entire course is for 40 hours (on a Sunday once in a month for 10 consecutive months).

- Candidates will be assessed on the basis of MCQs, roleplays, and practical assignments.

- At the end of the course, each candidate will be awarded a certificate.

\section{Eligibility for Program}

- Science graduate (B.Pharm, BSc/Associate of Science in Nursing or BSN, BA in Nutrition, or similar)

- Candidates must be working with a practicing or a diabetologist.

- Participants will be required to fill in the registration form and submit it at the center. The candidate selection is subject to fulfillment of the aforementioned criteria.

Program modules: There are 10 modules.

Participants are not to be charged any fees for the same.

Founded in: 1997; member of IDF since: 2011; number of members: $190{ }^{7}$

Other programs include VIVO diabetes educator program and 3-month online diabetes educators course.

\section{Association of Diabetes Educators}

$\mathrm{ADE}$ is a nonprofit organization comprising a team of trained diabetes nurse educators who are dedicated to provide teaching and counseling on diabetes management to health care professionals, people with diabetes, their family members, and any individual interested in understanding diabetes.

\section{Vision}

The vision is to improve life of people with diabetes through education.

\section{Mission}

The mission is to promote optimal health and well being for people with diabetes through effective education and counseling.

\section{Main Focus}

- To provide diabetes education and skills training

- To act as a consultant for diabetes education and training

- To provide a platform for diabetes health care professionals to network and seek peer support

- To facilitate opportunities for diabetes educators to participate in community outreach

\section{Activities}

Accreditation and Certification Board for Certified Diabetes Educators:

ADE through Journal of Diabetes Education as well as NDEP through Indian Diabetes Educators Journal are continuously doing the work of updating diabetes educators. ${ }^{8}$

\section{International Diabetes Federation}

The International Diabetes Federation (IDF) is an umbrella organization of more than 230 national diabetes associations in 170 countries and territories. It represents interests of the growing number of people with diabetes and those at risk. IDF has been leading the global diabetes community since 1950. The mission of the Association of Diabetes Educator Singapore (ADES) is to set the standard of excellence in the profession of diabetes education.

\section{Objectives of Certification}

- Providing a mechanism to demonstrate professional accomplishment and growth as a validation to self and working organization

- Providing formal recognition of specialty practice and knowledge

- Promoting continuing commitment to best practices, current standards, and knowledge in diabetes education

\section{Educational Seminars/Workshops}

- The aim is to create platforms for shared learning from various health care professionals and keep abreast of latest updates on diabetes treatment and management.

- Managing diabetes requires a concerted effort on the part of the patient. Knowledge alone is not sufficient to promote behavior change, and if the symptoms are absent, the motivation level goes down.

- This can be done by appointing a CDE in a diabetes clinic setup to ensure that the patient is well motivated till the next follow-up visit. ${ }^{9}$

\section{Technology Replacement}

Technology replacement focuses mainly on motivating patients through artificial intelligence that basically works as a diabetes educator and helps in self-management.

\section{Pros}

- Apps contain Storing data option where you can store your readings and Food log for better understanding and can help the physician prescribe medications. 
- Artificial intelligence or nutritional coaches to monitor your progress and encourage you throughout.

- Individualized goal, meal plans.

\section{Cons}

- It can be time consuming for patients as they have to keep entering the data daily.

- It lacks privacy.

- Things can be done without paying for the apps. Can manually fill the Glucose monitoring and Food logs in a book.

\section{Conclusion}

Health care is now focused on patient and public involvement to get better outcomes; thus, there needs to be proper involvement of both the patient and CDE. It therefore makes it a patient-centric method. Diabetes educators need to collaborate well with other health care professionals. Health care providers may fall short of time, and so with the help of a diabetes educator, patient self-management knowledge and personalized individual plans can be given, which will benefit the patient in a long run. Thus, a CDE will help improve patient outcomes.

\section{Conflict of Interest}

None.

\section{Acknowledgment}

The authors would like to thank Mr. Aslam Deshmukh from USV Ltd and Dr. Sanjay Kalra for their contribution to this work.

\section{References}

1 Cho NH, Shaw JE, Karuranga S, et al. IDF Diabetes Atlas: global estimates of diabetes prevalence for 2017 and projections for 2045. Diabetes Res Clin Pract 2018;138:271-281

2 Sridharan SG, Chittem M, Muppavaram N. A review of literature on diabetes self-management: scope for research and practice in India. J Soc Health Diabetes 2016;4:108-114

3 Deo MG. Doctor population ratio for India-the reality. Indian J Med Res 2013;137(4):632-635

4 Barnett DM, Joslin PE. A Centennial Portrait. Joslin Diabetes Center; 1998. Available at: https://www.joslin.org/about/ from_wandering_nurse_to_certified_diabetes_educator.html

5 Allen NA. The Diabetes Educator in the History of Diabetes Nursing, 1914-1936, 2003;29:976-989

6 National Certification Board of Diabetes Educators. https:// www.ncbde.org/. Accessed October 7, 2018

7 Joshi S, Joshi SR, Mohan V. Methodology and feasibility of a structured education program for diabetes education in India: the National Diabetes Educator Program. Indian J Endocrinol Metab 2013;17(3):396-401

8 Association of Diabetes Educators. http://www.diabeteseducatorsindia.com. Accessed October 14, 2018

9 International Diabetes Federation-Home. Idf.org. Retrieved October 14, 2018 